\title{
Equivalence operators in nilpotent systems
}

\author{
J. Dombi ${ }^{\text {a }}$, O. Csiszár ${ }^{\mathrm{b}, *}$ \\ ${ }^{a}$ University of Szeged, Institute of Informatics, Hungary \\ b Óbuda University, Institute of Applied Mathematics, Hungary
}

Received 5 April 2015; received in revised form 13 August 2015; accepted 14 August 2015

Available online 20 August 2015

\begin{abstract}
A consistent connective system generated by nilpotent operators is not necessarily isomorphic to the Eukasiewicz system. Using more than one generator function, consistent nilpotent connective systems (so-called bounded systems) can be obtained with the advantage of three naturally derived negation operators and thresholds. In this paper, equivalences in bounded systems are examined. Here, three different types of operators are studied, and a paradox of the equivalence (i.e. there is no equivalence relation in a non-Boolean setting which fulfils $\forall x e(x, x)=1$ and $e(x, n(x))=0)$ is resolved by aggregating the implication-based equivalence and its dual operator. We will also show that the aggregated equivalence has nice properties like associativity, threshold transitivity and T-transitiviy.
\end{abstract}

(c) 2015 Elsevier B.V. All rights reserved.

Keywords: Equivalence; Nilpotent operator; Łukasiewicz operator system; Bounded system; T-transitivity; Associativity

\section{Introduction}

The theory of fuzzy relations is a generalization of that of crisp relations of a set. Zadeh introduced the concept of fuzzy relations in [28] and the concept of fuzzy similarity relations in [29]. Since then, many authors studied fuzzy equivalence relations $[6,7,22,23]$ and it has proven to be useful in different contexts such as fuzzy control, approximate reasoning and fuzzy cluster analysis.

As shown by Gupta and Gupta [18], the condition $\mu(x, x)=1$ for $\forall x \in X$ is too strong for defining a fuzzy reflexive relation $\mu$ on a set $X$ (see also [27] and [8]). Therefore, new types of fuzzy reflexive relations were needed to be introduced. In [27], the concepts of $\epsilon$-reflexive fuzzy relations and weakly reflexive fuzzy relations were defined by weakening the standard reflexive fuzzy relation to $\mu(x, x) \geq \epsilon>0$. Gupta and Gupta [18] introduced G-reflexive fuzzy relations as a generalization of reflexive fuzzy relations.

While discussing fuzzy transitive relations, different approaches have been adopted. The first type of transitivity is that introduced by Zadeh [29], and the second type of transitivity is the so-called T-transitivity of fuzzy relations,

\footnotetext{
* Corresponding author.

E-mail addresses: dombi@inf.u-szeged.hu (J. Dombi), csiszar.orsolya @nik.uni-obuda.hu (O. Csiszár).
} 
defined with the help of the t-norm. In [4,5,10,11], fuzzy T-transitivity has been deeply studied. Recently, Mesiar et al. [21] have noticed that the associativity of a t-norm is superfluous in the above context, especially since we never have to aggregate more than two arguments. Thus, they have substituted a conjunctor instead of a t-norm. An alternative approach based on implications has been considered in [25,26]. In [19], I-transitivity, where the implicator I is nothing more than a binary operator satisfying the boundary conditions of an implication, was studied. Another type of transitivity, the so-called $\epsilon$-fuzzy transitivity, has been introduced in [3]. In [1], the authors introduced the concept of $(\alpha, \beta)$-fuzzy reflexive relations, as a generalization of fuzzy reflexive relation as well as of fuzzy G-reflexive relations. More general types of fuzzy symmetric relation, a $(\alpha, \beta)$-fuzzy symmetric relation and $(\alpha, \beta)$-fuzzy transitive relations, were also studied. The concepts of $(\alpha, \beta)$-fuzzy reflexive, symmetric and transitive relations naturally lead to the concept of $(\alpha, \beta)$-fuzzy equivalence relations on a set. In [9], the concept of a T-partition was introduced as a generalization of that of a classical partition.

Although the mentioned list of authors is by no means complete, it gives us a slight idea about the importance of the concept of fuzzy equivalence relations in different contexts. In our work we resolve a paradox of the equivalence (i.e. there is no equivalence relation in a non-Boolean setting which fulfils $\forall x e(x, x)=1$ and $e(x, n(x))=0)$ by aggregating the implication-based equivalence and its dual operator.

In our previous article [14], we showed that a consistent connective system generated by nilpotent operators is not necessarily isomorphic to the Łukasiewicz system. Using more than one generator function, consistent nilpotent connective systems can be obtained in a significantly different way with three naturally derived negation operators. As the class of non-strict t-norms has preferable properties that make them useful in constructing logical structures, the advantages of such systems are obvious [20]. Due to the fact that all continuous Archimedean (i.e. representable) nilpotent t-norms are isomorphic to the Łukasiewicz t-norm [17], the nilpotent systems studied earlier were all isomorphic to the well-known Łukasiewicz logic. Those consistent nilpotent connective systems which are not isomorphic to Łukasiewicz logic are called bounded systems (referring to the fact that the generators are bounded functions) [14]. Based on the results of [14] and [15], we now focus on equivalences in bounded systems.

The paper is organized as follows. After some preliminaries in Section 2, we define and examine the implicationbased equivalences in bounded systems in Section 3. Next, we introduce and examine the so-called dual equivalences in Section 4. Using the arithmetic mean operator examined in Section 5, the aggregated equivalences are introduced and studied in Section 6. We show that unlike the other two types, the aggregated equivalences are threshold transitive and associative as well. In Section 7, for further applications in image processing, the overall equivalence of two grey level images is defined and an important semantic meaning of the aggregated equivalences is given. Finally, in Section 7, we summarize our key results.

\section{Preliminaries}

First we recall the basic notations and results regarding equivalences and nilpotent systems.

\subsection{Equivalences}

There exist several approaches to the definition of equivalences. Equivalences can be considered as binary relations $[4,6-8,22,23]$.

Now we consider an equivalence as a connective. We give the definition of an equivalence as a binary operation on the unit interval according to Fodor and Roubens.

Definition 1. (See [16].) A function $e:[0,1]^{2} \rightarrow[0,1]$ is called equivalence if it satisfies the following conditions:

1. Symmetry, i.e. $e(x, y)=e(y, x)$ for $\forall x, y \in[0,1]$,

2. Compatibility, i.e. $e(0,1)=e(1,0)=0$ and $e(0,0)=e(1,1)=1$,

3. Reflexivity, i.e. $e(x, x)=1$ for $\forall x \in[0,1]$,

4. Monotonicity, i.e. $x \leq x^{\prime} \leq y^{\prime} \leq y \Rightarrow e(x, y) \leq e\left(x^{\prime}, y^{\prime}\right)$. 
Definition 2. An operator $e(x, y):[0,1]^{2} \rightarrow[0,1]$ is said to be

1. T-transitive with respect to a t-norm $T$, if $\forall x, y, z \in[0,1]: T(e(x, y), e(y, z)) \leq e(x, z)$,

2. threshold transitive with respect to a threshold $v(0<v<1)$, if $e(x, y) \geq v$ and $e(y, z) \geq v$ together imply $e(x, z) \geq v$ for $\forall x, y, z \in[0,1]$,

3. invariant with respect to a negation $n$, if $e(x, y)=e(n(x), n(y)) \forall x, y \in[0,1]$,

4. associative, if $e(x, e(y, z))=e(e(x, y), z)$ holds for $\forall x, y, z \in[0,1]$.

\subsection{Bounded systems}

To construct a logical system, we need to define the logical operators. As in [14] and [15], we will consider connective systems where the conjunction and the disjunction are special types of t-norms and t-conorms, respectively.

Definition 3. (See [14].) The triple $(c, d, n)$, where $c$ is a continuous Archimedean t-norm, $d$ is a continuous Archimedean $\mathrm{t}$-conorm and $n$ is a strong negation, is called a connective system.

Definition 4. (See [14].) A connective system is nilpotent if the conjunction $c$ is a nilpotent t-norm, and the disjunction $d$ is a nilpotent t-conorm.

Definition 5. (See [14].) Two connective systems, $\left(c_{1}, d_{1}, n_{1}\right)$ and $\left(c_{2}, d_{2}, n_{2}\right)$ are isomorphic, if there exists a monotonic bijection $\phi:[0,1] \rightarrow[0,1]$ such that

$$
\begin{aligned}
& \phi^{-1}\left(c_{1}(\phi(x), \phi(y))\right)=c_{2}(x, y) \\
& \phi^{-1}\left(d_{1}(\phi(x), \phi(y))\right)=d_{2}(x, y) \\
& \phi^{-1}\left(n_{1}(\phi(x))\right)=n_{2}(x) .
\end{aligned}
$$

Definition 6. (See $[14,24]$.) Let us define the cutting operation [ ] by

$$
[x]= \begin{cases}0 & \text { if } x<0 \\ x & \text { if } 0 \leq x \leq 1 \\ 1 & \text { if } 1<x\end{cases}
$$

and let the notation [ ] also act as brackets when writing the argument of an operator, so that we can write $f[x]$ instead of $f([x])$.

Definition 7. (See [14].) A connective system is called Łukasiewicz system if it is isomorphic to ([x+y-1], $[x+y], 1-x)$, i.e. if there exists a monotonic bijection $\phi:[0,1] \rightarrow[0,1]$ such that the connective system has the form

$$
\left(\phi^{-1}[\phi(x)+\phi(y)-1], \phi^{-1}[\phi(x)+\phi(y)], \phi^{-1}(1-\phi(x))\right) .
$$

Since the additive generator functions of the nilpotent t-norms and t-conorms are bounded and determined up to a multiplicative constant, they can be normalized (see [14]). Let us use the following notations for the uniquely defined normalized generator functions:

$$
f_{c}(x):=\frac{t(x)}{t(0)}, \quad f_{d}(x):=\frac{s(x)}{s(1)} .
$$

Using this concept, we have $f_{c}, f_{d}, f_{n}:[0,1] \rightarrow[0,1]$, where $f_{n}$ is the generator function of the negation used in our system.

Definition 8. (See [14].) The negations $n_{c}$ and $n_{d}$ generated by $f_{c}$ and $f_{d}$ respectively,

$$
n_{c}(x)=f_{c}^{-1}\left(1-f_{c}(x)\right)
$$


and

$$
n_{d}(x)=f_{d}^{-1}\left(1-f_{d}(x)\right)
$$

are called natural negations of $c$ and $d$ respectively.

Next, we recall certain key properties of connective systems and then give the propositions describing the conditions that a logical system must satisfy in order to have the above properties.

Definition 9. (See [14].) Classification property means that the law of contradiction holds, i.e.

$$
c(x, n(x))=0 \quad \forall x \in[0,1],
$$

and the excluded middle principle holds as well, i.e.

$$
d(x, n(x))=1 \quad \forall x \in[0,1] .
$$

Definition 10. (See [14].) The De Morgan identity means that

$$
c(n(x), n(y))=n(d(x, y))
$$

or

$$
d(n(x), n(y))=n(c(x, y)) \quad \forall x, y \in[0,1] .
$$

Remark 1. These two forms of the De Morgan law are equivalent, if the negation is involutive. The first De Morgan law holds with a strict negation $n$ if and only if the second holds with $n^{-1}[16]$.

Definition 11. (See [14].) A connective system is said to be consistent if the classification property (Definition 9) and the De Morgan identity (Definition 10) hold.

Proposition 1. (See [14] and also [16] 1.5.4. and 1.5.5., and [2] 2.3.12. and 2.3.15.) In a connective system (c, $d, n)$, the classification property holds if and only if $n_{d}(x) \leq n(x) \leq n_{c}(x)$, where $n_{c}$ and $n_{d}$ are the natural negations of $c$ and $d$ respectively.

Proposition 2. (See [14].) If $f_{c}$ is the normalized generator function of a conjunction in a nilpotent connective system, $f_{d}$ is a normalized generator function of the disjunction and $n$ is a strong negation, then the following statements are equivalent:

1. The De Morgan law holds in the connective system. That is,

$$
c(n(x), n(y))=n(d(x, y)) \quad \forall x, y \in[0,1] .
$$

2. The normalized generator functions of the conjunction, disjunction and negation operator obey the following equations (which are obviously equivalent to each other):

$$
\begin{aligned}
& n(x)=f_{c}^{-1}\left(f_{d}(x)\right)=f_{d}^{-1}\left(f_{c}(x)\right), \\
& f_{c}(x)=f_{d}(n(x)) \quad \text { or equivalently } f_{d}(x)=f_{c}(n(x)) .
\end{aligned}
$$

Proposition 3. (See [14].)

1. If the nilpotent connective system $(c, d, n)$ is consistent, then $f_{c}(x)+f_{d}(x) \geq 1$ for any $x \in[0,1]$, where $f_{c}$ and $f_{d}$ are the normalized generator functions of the conjunction $c$ and the disjunction $d$, respectively.

2. If $f_{c}(x)+f_{d}(x) \geq 1$ for any $x \in[0,1]$ and the De Morgan law holds, then the connective system $(c, d, n)$ satisfies the classification property as well (which now means that the system is consistent). 
The following proposition shows that a consistent nilpotent connective system is isomorphic to Łukasiewicz system if and only if the negations coincide.

Proposition 4. (See [14].) In a nilpotent connective system, $f_{c}(x)+f_{d}(x)=1$ if and only if

$$
n_{c}(x)=n_{d}(x) \text {. }
$$

Definition 12. (See [14].) A nilpotent connective system is called a bounded system, if

$$
\left.f_{c}(x)+f_{d}(x)>1 \quad \text { (or equivalently } n_{d}(x)<n(x)<n_{c}(x)\right)
$$

holds for all $x \in(0,1)$, where $f_{c}$ and $f_{d}$ are the normalized generator functions of the conjunction and disjunction, and $n_{c}, n_{d}$ are the natural negations.

Remark 2. (See [14].) Note that Łukasiewicz system is characterized by $n_{d}(x)=n_{c}(x)$; or equivalently,

$$
f_{c}(x)+f_{d}(x)=1 .
$$

Proposition 5. (See [15].) In a nilpotent connective system $(c, d, n)$ the residual implication has the following form.

$$
i_{R}(x, y)=f_{c}^{-1}\left[f_{c}(y)-f_{c}(x)\right],
$$

where $f_{c}$ is the generator function of $c$, and [ ] is the cutting operator defined in Definition 6.

In a nilpotent connective system $(c, d, n)$, we can define different types of S-implications.

Definition 13. (See [15].) The S-implications in a nilpotent connective system $(c, d, n)$ are defined as follows.

1. $i_{S_{n}}(x, y)=d(n(x), y), \quad x, y \in[0,1]$,

2. $i_{S_{d}}(x, y)=d\left(n_{d}(x), y\right), \quad x, y \in[0,1]$,

3. $i_{S_{c}}(x, y)=d\left(n_{c}(x), y\right), \quad x, y \in[0,1]$,

where $n_{c}$ and $n_{d}$ are the natural negations of $c$ and $d$, respectively.

Definition 14. (See [15].) In a nilpotent connective system $(c, d, n)$

1. $i_{S_{n}}^{c}(x, y)=n(c(x, n(y))), \quad x, y \in[0,1]$,

2. $i_{S_{d}}^{c^{n}}(x, y)=n_{d}\left(c\left(x, n_{d}(y)\right)\right), \quad x, y \in[0,1]$,

3. $i_{S_{c}^{c}}^{c^{d}}(x, y)=n_{c}\left(c\left(x, n_{c}(y)\right)\right), \quad x, y \in[0,1]$,

where $n_{c}$ and $n_{d}$ are the natural negations of $c$ and $d$, respectively.

Proposition 6. (See [15].) In a nilpotent connective system $(c, d, n)$

1. $i_{S_{n}}(x, y)=f_{d}^{-1}\left[f_{c}(x)+f_{d}(y)\right]$,

2. $i_{S_{d}}(x, y)=f_{d}^{-1}\left[1-f_{d}(x)+f_{d}(y)\right]$,

3. $i_{S_{c}}(x, y)=f_{d}^{-1}\left[f_{d}(y)+f_{d}\left(n_{c}(x)\right)\right]$,

where $f_{c}$ and $f_{d}$ are the normalized generator functions of $c$ and $d$, respectively.

Proposition 7. (See [15].) In a nilpotent connective system $(c, d, n) i_{S_{c}}^{c}(x, y)=f_{c}^{-1}\left[f_{c}(y)-f_{c}(x)\right]=i_{R}(x, y)$, where $f_{c}$ is the normalized generator function of $c$.

Proposition 8. (See [15].) In a nilpotent connective system $(c, d, n)$, any two of the implications defined so far coincide if and only if $f_{c}(x)+f_{d}(x)=1$, where $f_{c}$ and $f_{d}$ are the normalized generator functions of $c$ and $d$, respectively. 
Table 1

Rational generator functions.

\begin{tabular}{lllll}
\hline & $f(x)$ (generator) & $f^{-1}(x)$ & $1-f(x)$ & Negation \\
\hline Negation & $\frac{1}{1+\frac{v}{1-v} \frac{1-x}{x}}$ & $\frac{1}{1+\frac{1-v}{v} \frac{1-x}{x}}$ & $\frac{1}{1+\frac{1-v}{v} \frac{x}{1-x}}$ & $n(x)=\frac{1}{1+\left(\frac{1-v}{v}\right)^{2} \frac{x}{1-x}}$ \\
Conjunction & $\frac{1}{1+\frac{v_{c}}{1-v_{c}} \frac{x}{1-x}}$ & $\frac{1}{1+\frac{v_{c}}{1-v_{c}} \frac{x}{1-x}}$ & $\frac{1}{1+\frac{1-v_{c}}{v_{c}} \frac{1-x}{x}}$ & $n_{c}(x)=\frac{1}{1+\left(\frac{1-v_{c}}{v_{c}}\right)^{2} \frac{x}{1-x}}$ \\
Disjunction & $\frac{1}{1+\frac{v_{d}}{1-v_{d}} \frac{1-x}{x}}$ & $\frac{1}{1+\frac{1-v_{d}}{v_{d}} \frac{1-x}{x}}$ & $\frac{1}{1+\frac{1-v_{d}}{v_{d}} \frac{x}{1-x}}$ & $n_{d}(x)=\frac{1}{1+\left(\frac{1-v_{d}}{v_{d}}\right)^{2} \frac{x}{1-x}}$ \\
\hline
\end{tabular}

\subsection{Rational generator functions}

Next, we consider the case of the rational family of the normalized generator functions (see Table 1) introduced by Dombi in [12]. In the following sections, we will use these functions in the examples to illustrate our results.

Proposition 9. (See [14].) For the Dombi functions

$$
\begin{array}{ll}
f_{n}(x)=\frac{1}{1+\frac{v}{1-v} \frac{1-x}{x}}, & f_{n}(0)=0, v \in(0,1), \\
f_{c}(x)=\frac{1}{1+\frac{v_{c}}{1-v_{c}} \frac{x}{1-x}}, & f_{d}(0)=0, v_{c} \in(0,1), \\
f_{d}(x)=\frac{1}{1+\frac{v_{d}}{1-v_{d}} \frac{1-x}{x}}, & f_{c}(1)=0, v_{d} \in(0,1),
\end{array}
$$

the following statements are equivalent:

1. The connective system generated by the Dombi functions in Proposition 9 satisfies the De Morgan law.

2. For parameters $v_{d}$ and $v_{c}$ in the normalized generator functions and for parameter $v$ in the negation function the following equation holds:

$$
\left(\frac{1-v}{v}\right)^{2}=\frac{v_{c}}{1-v_{c}} \frac{1-v_{d}}{v_{d}} .
$$

Remark 3. Note that the fixpoints of the negation operators $n, n_{c}$ and $n_{d}$ are $v, 1-v_{c}$ and $v_{d}$ respectively.

\section{Equivalences in bounded systems}

Let us now consider a nilpotent connective system $(c, d, n)$ and let us denote the normalized generator functions of $c$ and $d$ by $f_{c}$ and $f_{d}$, respectively. Using the above-defined implications $i_{c}$ and $i_{d}$, we can define two different types of equivalences.

Definition 15. The conjunctive and disjunctive equivalence operators (see Fig. 1) are defined as follows.

$$
\begin{aligned}
& e_{c}(x, y)=c\left(i_{c}(x, y), i_{c}(y, x)\right) \\
& e_{d}(x, y)=n_{d}\left(d\left(n_{d}\left(i_{d}(x, y)\right), n_{d}\left(i_{d}(y, x)\right)\right)\right)
\end{aligned}
$$

Proposition 10. In a bounded system,

$$
e_{c}(x, y)=f_{c}^{-1}\left[\left|f_{c}(x)-f_{c}(y)\right|\right]
$$

and similarly,

$$
e_{d}(x, y)=f_{d}^{-1}\left[1-\left|f_{d}(x)-f_{d}(y)\right|\right] .
$$




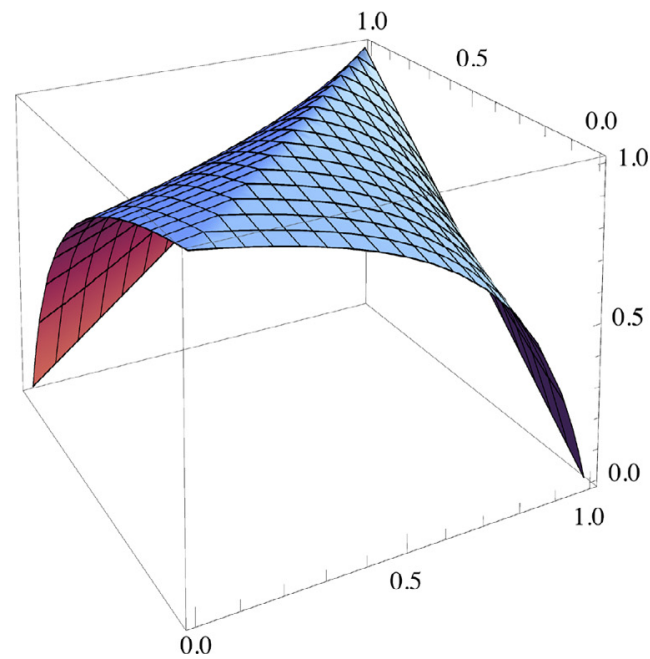

(a) $e_{c}$ with $\nu_{c}=0.3$

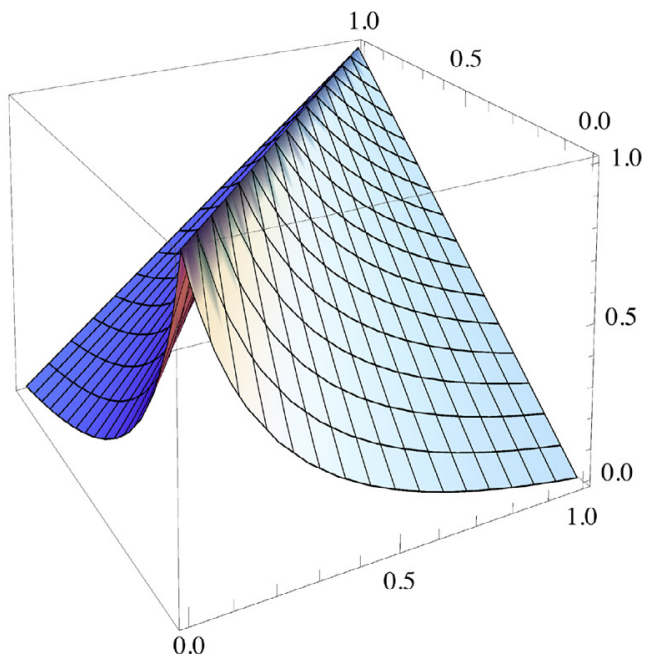

(b) $e_{d}$ with $\nu_{d}=0.3$

Fig. 1. $e_{c}(x, y)$ and $e_{d}(x, y)$ for rational generators.

\section{Proof.}

$$
e_{c}(x, y)=f_{c}^{-1}\left[\left[f_{c}(y)-f_{c}(x)\right]+\left[f_{c}(x)-f_{c}(y)\right]\right] .
$$

If $x<y$, then $f_{c}(x) \geq f_{c}(y)$, which means that we have $f_{c}^{-1}\left[f_{c}(x)-f_{c}(y)\right]$. Similarly, if $y>x$, then $f_{c}(x) \leq f_{c}(y)$ and we get $f_{c}^{-1}\left[f_{c}(y)-f_{c}(x)\right]$. Similarly for $e_{d}$, by using $n_{d}\left(i_{d}(y, x)\right)=f_{d}^{-1}\left[f_{d}(y)-f_{d}(x)\right]$, we obtain

$$
n_{d}\left(e_{d}(x, y)\right)=f_{d}^{-1}\left[\left[f_{d}(x)-f_{d}(y)\right]+\left[f_{d}(y)-f_{d}(x)\right]\right]=f_{d}^{-1}\left[\left|f_{d}(x)-f_{d}(y)\right|\right] .
$$

Therefore,

$$
e_{d}(x, y)=f_{d}^{-1}\left[1-\left|f_{d}(x)-f_{d}(y)\right|\right] .
$$

Remark 4. Since $0 \leq\left|f_{c}(x)-f_{c}(y)\right| \leq 1$ and $0 \leq 1-\left|f_{d}(x)-f_{d}(y)\right| \leq 1$, the cutting function can be omitted here. For conceptual reasons, we prefer to leave it in all of the formulae.

\subsection{Properties of $e_{c}(x, y)$ and $e_{d}(x, y)$}

Next, we will examine the chief properties of $e_{c}(x, y)$ and $e_{d}(x, y)$ and show that they coincide if and only if the connective system is a Łukasiewicz system.

Proposition 11. Let $v_{c}$ and $v_{d}$ be the fixpoints of $n_{c}$ and $n_{d}$ respectively. The operators, $e_{c}(x, y)$ and $e_{d}(x, y)$ have the following properties:

\section{Compatibility (see Definition 1).}

2. Symmetry (see Definition 1).

3. Reflexivity (see Definition 1).

4. Monotonicity (see Definition 1).

5. $e_{c}$ is T-transitive with respect to the conjunction $c$ (see Definition 2) and similarly, $e_{d}$ is T-transitive with respect to the $t$-norm generated by $1-f_{d}(x)$.

6. $e_{c}$ and $e_{d}$ are not threshold transitive (see Definition 2) with respect to $v_{c}$ and $v_{d}$.

7. Invariance (see Definition 2) with respect to $n_{c}$ and $n_{d}$.

8. $e_{c}(1, x)=e_{c}(1, x)=x, e_{d}(0, x)=n_{d}(x)$, and similarly, $e_{c}(0, x)=n_{c}(x)$.

9. $e_{c}(x, y)=0$ if and only if $x, y \in\{0,1\}$ and $x \neq y$. Similarly, $e_{d}(x, y)=0$ if and only if $x, y \in 0,1$ and $x \neq y$. 
10. $n_{d}\left(e_{d}(x, y)\right)=e_{d}\left(n_{d}(x), y\right)$ if and only if $x \in\{0,1\}$ or $y \in\{0,1\}$ and $n_{c}\left(e_{c}(x, y)\right)=e_{c}\left(n_{c}(x), y\right)$ if and only if $x \in\{0,1\}$ or $y \in\{0,1\}$.

11. $e_{c}\left(x, v_{c}\right) \geq v_{c}$ and similarly, $e_{d}\left(x, v_{d}\right) \geq v_{d}$.

\section{Proof.}

1. From $f_{c}^{-1}(0)=1$, it follows that $e_{c}(1,1)=e_{c}(0,0)=1$. From $f_{c}(1)=0, f_{c}(0)=1$ and $f_{c}^{-1}(1)=0$, we get that $e_{c}(0,1)=e_{c}(1,0)=0$. Similarly, from $f_{d}^{-1}(1)=1$, it follows that $e_{d}(1,1)=e_{d}(0,0)=1$. From $f_{d}(1)=1$, $f_{d}(0)=0$ and $f_{d}^{-1}(0)=0$, we get that $e_{d}(0,1)=e_{d}(1,0)=0$.

2. Trivial.

3. $e_{c}(x, x)=f_{c}^{-1}(0)=1$ and $e_{d}(x, x)=f_{d}^{-1}(1)=1$.

4. We have to show that from $x \leq x^{\prime} \leq y^{\prime} \leq y$ it follows that $e_{c}(x, y) \leq e_{c}\left(x^{\prime}, y^{\prime}\right)$. Using the monotonicity of $f_{c}(x)$ and $f_{c}^{-1}(x)$, the statement follows immediately. For $e_{d}$, we have to show that from $x \leq x^{\prime} \leq y^{\prime} \leq y$ it follows that $e_{d}(x, y) \leq e_{d}\left(x^{\prime}, y^{\prime}\right)$. Using the monotonicity of $f_{d}(x)$ and $f_{d}^{-1}(x)$ the statement follows immediately.

5. By using the decreasing property of $f_{c}^{-1}$ and the triangle inequality, we obtain

$$
c(e(x, y), e(y, z))=f_{c}^{-1}\left(\left|f_{c}(x)-f_{c}(y)\right|+\left|f_{c}(y)-f_{c}(z)\right|\right) \leq f_{c}^{-1}\left(\left|f_{c}(x)-f_{c}(z)\right|\right)=e(x, z) .
$$

The proof is similar for $e_{d}$ as well.

6. $e_{c}(x, y) \geq v_{c}$ iff $\left|f_{c}(x)-f_{c}(y)\right| \leq \frac{1}{2}$ and similarly, $e_{c}(y, z) \geq v_{c}$ iff $\left|f_{c}(y)-f_{c}(z)\right| \leq \frac{1}{2}$. Obviously, these conditions are not sufficient for $\left|f_{c}(x)-f_{c}(z)\right| \leq \frac{1}{2}$. Similarly, $e_{d}(x, y) \geq v_{d}$ iff $1-\left|f_{d}(x)-f_{d}(y)\right| \geq \frac{1}{2}$ and similarly, $e_{d}(y, z) \geq v_{d}$ iff $\left|f_{d}(y)-f_{d}(z)\right| \geq \frac{1}{2}$. Obviously, these conditions are not sufficient for $1-\left|f_{d}(x)-f_{d}(z)\right| \geq \frac{1}{2}$.

7. $e_{c}\left(n_{c}(x), n_{c}(y)\right)=f_{c}^{-1}\left[\left|f_{c}\left(n_{c}(x)\right)-f_{c}\left(n_{c}(y)\right)\right|\right]=f_{c}^{-1}\left[\left|1-f_{c}(x)-\left(1-f_{c}(y)\right)\right|\right]=f_{c}^{-1}\left[\left|f_{c}(y)-f_{c}(x)\right|\right]=$ $e_{c}(x, y)$. Similarly, $e_{d}\left(n_{d}(x), n_{d}(y)\right)=f_{d}^{-1}\left[\left|f_{d}\left(n_{d}(x)\right)-f_{d}\left(n_{d}(y)\right)\right|\right]=f_{d}^{-1}\left[\left|1-f_{d}(x)-\left(1-f_{d}(y)\right)\right|\right]=$ $f_{d}^{-1}\left[\left|f_{d}(y)-f_{d}(x)\right|\right]=e_{d}(x, y)$.

8. Using the fact that $f_{c}(1)=0$, we get $e_{c}(1, x)=f_{c}^{-1}\left[\left|f_{c}(1)-f_{c}(x)\right|\right]=x$.

Similarly, using the fact that $f_{c}(0)=1$ and that $0 \leq f_{c}(x) \leq 1$ for $\forall x \in[0,1]$, we get $e_{c}(0, x)=f_{c}^{-1}\left[\mid f_{c}(0)-\right.$ $\left.f_{c}(x) \mid\right]=n_{c}(x)$. For $e_{d}$, using the fact that $f_{d}(1)=1$ and that $0 \leq f_{d}(x) \leq 1$ for $\forall x \in[0,1]$ we get $e_{d}(1, x)=$ $f_{d}^{-1}\left[1-\left|f_{d}(1)-f_{d}(x)\right|\right]=x$. From $f_{d}(0)=0$, we get $e_{d}(0, x)=f_{d}^{-1}\left[1-\left|f_{d}(0)-f_{d}(x)\right|\right]=n_{d}(x)$.

9. If $e_{c}(x, y)=0$, then $\left|f_{c}(x)-f_{c}(y)\right|=1$, from which $x, y \in 0,1$ and $x \neq y$. Going in the opposite direction is trivial.

10. $n_{c}\left(e_{c}(x, y)\right)=f_{c}^{-1}\left(1-\left|f_{c}(x)-f_{c}(y)\right|\right)$ and $e_{c}\left(n_{c}(x), y\right)=f_{c}^{-1}\left(\left|1-f_{c}(x)-f_{c}(y)\right|\right)$. Considering the four cases and using the monotonicity of $f_{c}(x)$, we get that $x \in\{0,1\}$ or $y \in\{0,1\}$. The proof is similar for $e_{d}(x, y)$ as well.

11. Using the monotonicity property of $f_{c}(x)$ and the fact that $f_{c}\left(v_{c}\right)=\frac{1}{2}$, we get $e_{c}\left(x, v_{c}\right)=f_{c}^{-1}\left[\mid f_{c}(x)-\right.$ $\left.f_{c}\left(v_{c}\right) \mid\right]=f_{c}^{-1}\left[\left|f_{c}(x)-\frac{1}{2}\right|\right] \geq v_{c}$, since $0 \leq\left[\left|f_{c}(x)-\frac{1}{2}\right|\right] \leq \frac{1}{2}$. Similarly, using the monotonicity property of $f_{d}(x)$ and the fact that $f_{d}\left(v_{d}\right)=\frac{1}{2}$, we get

$$
e_{d}\left(x, v_{d}\right)=f_{d}^{-1}\left[1-\left|f_{d}(x)-f_{d}\left(v_{d}\right)\right|\right]=f_{d}^{-1}\left[1-\left|f_{d}(x)-\frac{1}{2}\right|\right] \geq v_{d},
$$

since $\frac{1}{2} \leq 1-\left|f_{d}(x)-\frac{1}{2}\right| \leq 1$.

Proposition 12. If $x, y>v_{c}$ or $x, y<v_{c}$, then $e_{c}(x, y)>v_{c}$. Similarly, if $x, y>v_{d}$ or $x, y<v_{d}$, then $e_{d}(x, y)>v_{d}$.

Proof. If $x, y>v_{c}$, then $f_{c}(x), f_{c}(y)<\frac{1}{2}$, so $\left|f_{c}(x)-f_{c}(y)\right|<\frac{1}{2}$, which means that $e_{c}(x, y)>v_{c}$. Similarly, if $x, y<v_{c}$, then $f_{c}(x), f_{c}(y)>\frac{1}{2}$, so $\left|f_{c}(x)-f_{c}(y)\right|<\frac{1}{2}$, which means that $e_{c}(x, y)>v_{c}$. For $e_{d}$, if $x, y>v_{d}$, then $f_{d}(x), f_{d}(y)>\frac{1}{2}$, so $\left|f_{d}(x)-f_{d}(y)\right|<\frac{1}{2}$, which means that $e_{d}(x, y)>v_{d}$. Similarly, if $x, y<v_{d}$, then $f_{d}(x), f_{d}(y)<\frac{1}{2}$, so $\left|f_{d}(x)-f_{d}(y)\right|<\frac{1}{2}$, which means that $e_{d}(x, y)>v_{d}$.

Remark 5. $e_{c}$ and $e_{d}$ are not associative. 
Proof. A possible counterexample might be the case of rational generators with $v_{c}=0.6$ and $v_{d}=0.3, x=0.3$, $y=0.4$ and $y=0.5$. In this case we get $e_{c}\left(x, e_{c}(y, z)\right) \approx 0.39, e_{c}\left(e_{c}(x, y), z\right) \approx 0.62$, while for $e_{d}\left(x, e_{d}(y, z)\right) \approx 0.38$ and $e_{d}\left(e_{d}(x, y), z\right) \approx 0.64$.

Proposition 13. In a connective system the above-defined equivalences $e_{c}(x, y)$ and $e_{d}(x, y)$ coincide if and only if $f_{c}(x)+f_{d}(x)=1$ (or equivalently $n_{c}=n_{d}$, i.e. in a Eukasiewicz system), where $f_{c}$ and $f_{d}$ are the normalized generation function of the conjunction and disjunction operators, respectively.

\section{Proof.}

1. If $f_{c}(x)+f_{d}(x)=1$, then $f_{c}(x)=1-f_{d}(x)$ and $f_{c}^{-1}(x)=f_{d}^{-1}(1-x)$, from which we get $e_{c}(x, y)=$ $f_{c}^{-1}\left[\left|f_{c}(x)-f_{c}(y)\right|\right]=f_{d}^{-1}\left[1-\left|f_{d}(x)-f_{d}(y)\right|\right]=e_{d}(x, y)$.

2. If $e_{c}(x, y)=e_{d}(x, y)$, then in particular $e_{c}(0, x)=e_{d}(x, 0)$, which means that $n_{c}(x)=n_{d}(x)$ must hold for all $x \in[0,1]$.

\section{Dual equivalences}

In classical logic, the equivalence operator has the following important property as well: $e(x, n(x))=0$. As it is well known, demanding $\forall x e(x, x)=1$ and $e(x, n(x))=0$ at the same time in a non-Boolean setting, gives rise to a paradox.

Lemma 1. There is no equivalence relation which fulfils $\forall x e(x, x)=1$ and $e(x, n(x))=0$.

Proof. Let $v$ be the fixpoint of the negation $n(x)$. Then $1=e(v, v)=e(v, n(v))=0$, which is a contradiction.

However, in practical applications the property $e(x, n(x))=0$ might be of even greater importance than reflexivity [13]. Motivated by this demand, below we will define new types of operators.

First, we will define the so-called dual equivalence, denoted by $\bar{e}$. Let us now consider a nilpotent connective system $(c, d, n)$ and let us denote the normalized generator functions of $c$ and $d$ by $f_{c}$ and $f_{d}$, respectively.

Definition 16. The dual equivalence operations (see Fig. 2) are defined as follows.

$$
\begin{aligned}
& \bar{e}_{c}(x, y)=n_{c}\left(e_{c}\left(x, n_{c}(y)\right) \quad\right. \text { and } \\
& \bar{e}_{d}(x, y)=n_{d}\left(e_{d}\left(x, n_{d}(y)\right) .\right.
\end{aligned}
$$

Proposition 14. In a bounded system the equivalence operators have the form

$$
\begin{aligned}
& \bar{e}_{c}(x, y)=f_{c}^{-1}\left[1-\left|f_{c}(x)+f_{c}(y)-1\right|\right] \quad \text { and } \\
& \bar{e}_{d}(x, y)=f_{d}^{-1}\left[\left|f_{d}(x)+f_{d}(y)-1\right|\right] .
\end{aligned}
$$

Proof. The formulae can be derived from direct calculation.

Remark 6. Since $0 \leq\left|f_{c}(x)+f_{c}(y)-1\right| \leq 1$ and $0 \leq\left|f_{d}(x)+f_{d}(y)-1\right| \leq 1$, the cutting function can be omitted here. For conceptual reasons, we prefer to leave it in all of the formulae.

\subsection{Properties of $\bar{e}_{d}$ and $\bar{e}_{c}$}

Next, we will study the main properties of the dual equivalences.

Proposition 15. Let $v_{c}$ and $v_{d}$, be the fixpoints of $n_{c}$ and $n_{d}$, respectively. Then the operators $\bar{e}_{c}(x, y)$ and $\bar{e}_{d}(x, y)$ have the following properties: 


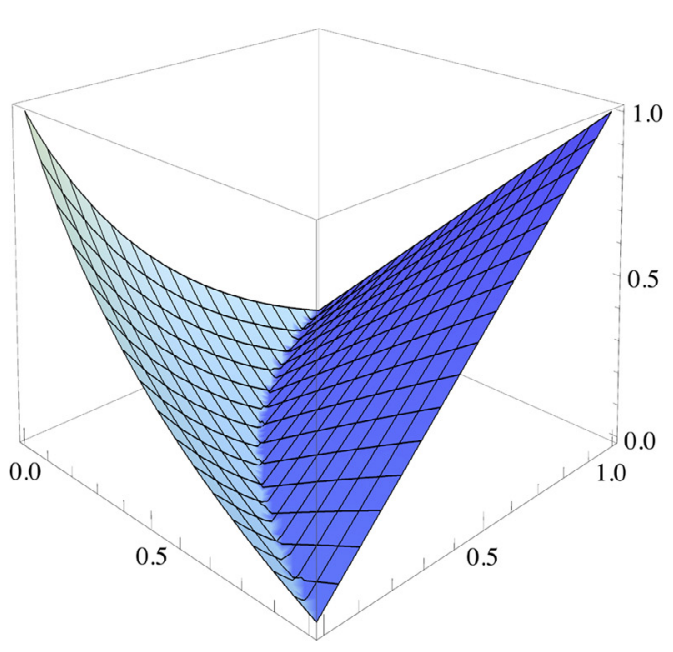

(a) $\bar{e}_{c}$ with $\nu_{c}=0.6$

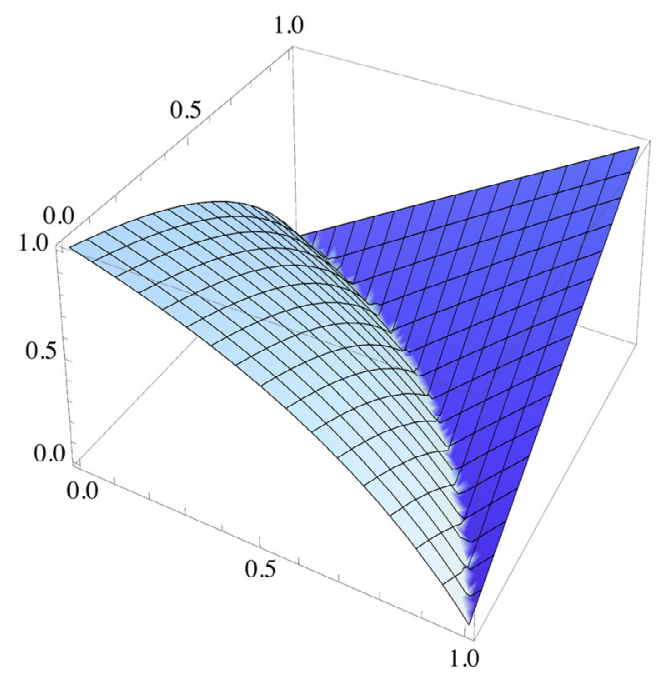

(b) $\bar{e}_{d}$ with $\nu_{d}=0.6$

Fig. 2. $\bar{e}_{c}(x, y)$ and $\bar{e}_{d}$ with rational generators.

\section{Compatibility (see Definition 1).}

2. Symmetry (see Definition 1).

3. $\bar{e}_{c}(x, y)$ and $\bar{e}_{d}(x, y)$ are not reflexive, but $\bar{e}_{c}\left(x, n_{c}(x)\right)=\bar{e}_{d}\left(x, n_{d}(x)\right)=0$.

4. $\bar{e}_{c}(x, y)$ and $\bar{e}_{d}(x, y)$ are not monotonic.

5. $\bar{e}_{c}$ is T-transitive with respect to the conjunction $c$ (see Definition 2) and similarly, $\bar{e}_{d}$ is T-transitive with respect to the t-norm generated by $1-f_{d}(x)$.

6. $\bar{e}_{c}(x, y)$ and $\bar{e}_{d}(x, y)$ are not threshold transitive with respect to $v_{c}$ and $v_{d}$ (see Definition 2).

7. Invariance with respect to $n_{c}$ and $n_{d}$ (see Definition 2 ).

8. $\bar{e}_{c}(1, x)=\bar{e}_{c}(1, x)=x$ $\bar{e}_{d}(0, x)=n_{d}(x)$, and similarly, $\bar{e}_{c}(0, x)=n_{c}(x)$.

9. $\bar{e}_{c}(x, y)=0$ if and only if $x=n_{c}(y)$ and similarly, $\bar{e}_{d}(x, y)=0$ if and obly if $x=n_{d}(y)$.

10. $n_{d}\left(\bar{e}_{d}(x, y)\right)=\bar{e}_{d}\left(n_{d}(x), y\right)$ if and only if $x \in\{0,1\}$ or $y \in\{0,1\}$ and $n_{c}\left(\bar{e}_{c}(x, y)\right)=\bar{e}_{c}\left(n_{c}(x), y\right)$ if and only if $x \in\{0,1\}$ or $y \in\{0,1\}$.

11. $\bar{e}_{c}\left(x, v_{c}\right) \leq v_{c}$ and $\bar{e}_{d}\left(x, v_{d}\right) \leq v_{d}$.

\section{Proof.}

1. Using the formulae given in Proposition 14, compatibility is trivial.

2. Using the formulae given in Proposition 14, symmetry is trivial as well.

3. Follows from direct calculation. Since $\bar{e}_{c}\left(x, n_{c}(x)\right)=0$ holds for the fixpoint $v_{c}$ of the $n_{c}$ as well, reflexivity cannot hold. Similarly for $\bar{e}_{d}$.

4. A counterexample might be the case of rational generators with $v_{c}=0.3 \cdot \bar{e}_{c}(0.1,0.6) \approx 0.75$, while $\bar{e}_{c}(0.4,0.5) \approx$ 0.68 , and similarly for $\bar{e}_{d}(0.4,0.6) \approx 0.21$, while $\bar{e}_{d}(0.45,0.5) \approx 0.19$.

5. By using the decreasing property of $f_{c}^{-1}$ and the fact that $|a+b-1|+|b+c-1|-1 \leq|a+c-1|$ holds for all $a, b, c \in[0,1]$, we obtain

$$
\begin{aligned}
c\left(\bar{e}_{c}(x, y), \bar{e}_{c}(y, z)\right) & =f_{c}^{-1}\left(2-\left|f_{c}(x)+f_{c}(y)-1\right|-\left|f_{c}(y)+f_{c}(z)-1\right|\right) \\
& \leq f_{c}^{-1}\left(1-\left|f_{c}(x)+f_{c}(z)-1\right|\right)=\bar{e}_{c}(x, z) .
\end{aligned}
$$

The proof is similar for $\bar{e}_{d}$ as well.

6. A possible counterexample might be for rational generators with $v_{c}=0.3, x=0.85, y=0.9$ and $z=0.87$, or for $v_{d}=0.3, x=0.7, y=0.9$ and $z=0.6$. 
7. $\bar{e}_{c}\left(n_{c}(x), n_{c}(y)\right)=1-f_{c}^{-1}\left[\left|1-f_{c}(x)+1-f_{c}(y)-1\right|\right]=\bar{e}_{c}(x, y)$ and similarly, $\bar{e}_{d}\left(n_{d}(x), n_{d}(y)\right)=f_{d}^{-1}[\mid 1-$ $\left.f_{d}(x)+1-f_{d}(y)-1 \mid\right]=\bar{e}_{d}(x, y)$.

8. Using the fact that $f_{c}(1)=0$, we get $\bar{e}_{c}(1, x)=f_{c}^{-1}\left[1-\left|f_{c}(1)+f_{c}(x)-1\right|\right]=x$.

Similarly, using the fact that $f_{c}(0)=1$ and that $0 \leq f_{c}(x) \leq 1$ for $\forall x \in[0,1]$, we get $\bar{e}_{c}(0, x)=f_{c}^{-1}\left[1-\mid f_{c}(0)+\right.$ $\left.f_{c}(x)-1 \mid\right]=n_{c}(x)$. For $e_{d}$, using the fact that $f_{d}(1)=1$ and that $0 \leq f_{d}(x) \leq 1$ for $\forall x \in[0,1]$ we get $\bar{e}_{d}(1, x)=$ $f_{d}^{-1}\left[\left|f_{d}(1)+f_{d}(x)-1\right|\right]=x$. Using the fact that $f_{d}(0)=0$, we get $\bar{e}_{d}(0, x)=f_{d}^{-1}\left[\left|f_{d}(0)-f_{d}(x)-1\right|\right]=$ $n_{d}(x)$.

9. Using the fact that $f_{c}\left(n_{c}(x)\right)=1-f_{c}(x)$ and $f_{d}\left(n_{d}(x)\right)=1-f_{d}(x)$, we get $\bar{e}_{c}\left(x, n_{c}(x)\right)=1-f_{c}^{-1}(0)=0$ and similarly $\bar{e}_{d}\left(x, n_{d}(x)\right)=f_{d}^{-1}(0)=0$. If $\bar{e}_{c}(x, y)=0$, then $f_{c}(x)+f_{c}(y)=1$, from which $f_{c}(x)=1-f_{c}(y)$, i.e. $x=f_{c}^{-1}\left[1-f_{c}(y)\right]=n_{c}(y)$. Similarly, if $\bar{e}_{d}(x, y)=0$, then $f_{d}(x)+f_{d}(y)=1$, from which $f_{d}(x)=1-f_{d}(y)$, i.e. $x=f_{d}^{-1}\left[1-f_{d}(y)\right]=n_{d}(y)$.

10. $n_{c}\left(\bar{e}_{c}(x, y)\right)=f_{c}^{-1}\left(1-\left|f_{c}(x)+f_{c}(y)-1\right|\right)$ and $\bar{e}_{c}\left(n_{c}(x), y\right)=f_{c}^{-1}\left(1-\left|f_{c}(x)-f_{c}(y)\right|\right)$. Considering the four cases and using the monotonicity of $f_{c}(x)$, we get that $x \in\{0,1\}$ or $y \in\{0,1\}$. The proof for $e_{d}(x, y)$ follows in a similar way.

11. Using the strict monotonicity of $f_{c}, f_{d}$ and their inverse functions, and the fact that $f_{c}\left(v_{c}\right)=f_{d}\left(v_{d}\right)=\frac{1}{2}$, the proof can be found by direct calculation.

Remark 7. $\bar{e}_{c}(x, y)$ and $\bar{e}_{d}(x, y)$ are not associative.

Proof. It is easy to find a counterexample, e.g. for rational generators with $v_{c}=0.3, \bar{e}_{c}\left(0.3, \bar{e}_{c}(0.4,0.5)\right) \approx 0.58$, while $\bar{e}_{c}\left(\bar{e}_{c}(0.3,0.4), 0.5\right) \approx 0.16$. Similarly, $\bar{e}_{d}\left(0.1, \bar{e}_{d}(0.5,0.7)\right) \approx 0.12$, while $\bar{e}_{d}\left(\bar{e}_{c}(0.1,0.5), 0.7\right) \approx 0.03$.

Proposition 16. In a connective system the above-defined equivalences $\bar{e}_{c}(x, y)$ and $\bar{e}_{d}(x, y)$ coincide if and only if $f_{c}(x)+f_{d}(x)=1$ (or equivalently $n_{c}=n_{d}$, i.e. in a Eukasiewicz system), where $f_{c}$ and $f_{d}$ are the normalized generation function of the conjunction and disjunction operators, respectively.

\section{Proof.}

1. If $f_{c}(x)+f_{d}(x)=1$, then $f_{c}(x)=1-f_{d}(x)$ and $f_{c}^{-1}(x)=f_{d}^{-1}(1-x)$, from which we get $\bar{e}_{c}(x, y)=$ $f_{c}^{-1}\left[1-\left|f_{c}(x)+f_{c}(y)-1\right|\right]=f_{d}^{-1}\left[\left|1-f_{d}(x)-f_{d}(y)\right|\right]=e_{d}(x, y)$.

2. If $e_{c}(x, y)=e_{d}(x, y)$, then in particular $\bar{e}_{c}(0, x)=\bar{e}_{d}(x, 0)$, which means that $n_{c}(x)=n_{d}(x)$ must hold for all $x \in[0,1]$.

\section{Arithmetic mean operators in bounded systems}

Let us define the so-called arithmetic mean operators in a bounded system.

Definition 17. In a connective system $(c, d, n)$

$$
m_{c}^{(\alpha)}(x, y):=f_{c}^{-1}\left[\alpha \cdot f_{c}(x)+(1-\alpha) \cdot f_{c}(y)\right]
$$

and similarly,

$$
m_{d}^{(\alpha)}(x, y):=f_{d}^{-1}\left[\alpha \cdot f_{d}(x)+(1-\alpha) \cdot f_{d}(y)\right],
$$

where $f_{c}$ and $f_{d}$ are the normalized generator functions of the conjunction and disjunction operators, respectively, $0<\alpha<1 . m_{c}$ and $m_{d}$ are called weighted arithmetic mean operators.

Proposition 17. $m_{c}^{(\alpha)}(x, y)$ and $m_{d}^{(\alpha)}(x, y)$ satisfy the self-De Morgan property with respect to $n_{c}$ and $n_{d}$ respectively, i.e.

$$
n_{c}\left(m_{c}^{(\alpha)}(x, y)\right)=m_{c}^{(\alpha)}\left(n_{c}(x), n_{c}(y)\right)
$$




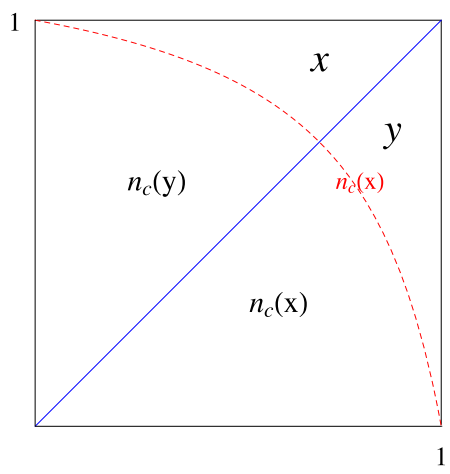

(a) $e_{c}^{*}$

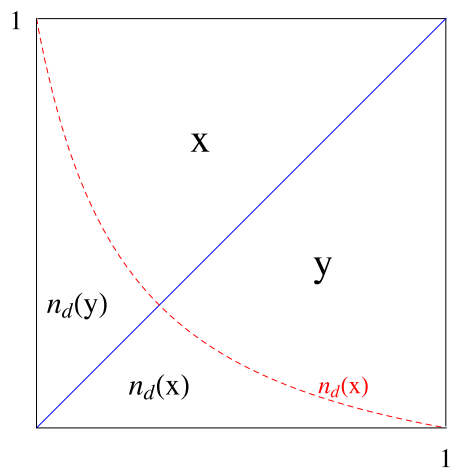

(b) $e_{d}^{*}$

Fig. 3. The domain of aggregated equivalences.

and similarly,

$$
n_{d}\left(m_{d}^{(\alpha),}(x, y)\right)=m_{d}^{(\alpha),}\left(n_{d}(x), n_{d}(y)\right) .
$$

\section{Proof.}

$$
\begin{aligned}
n_{c}\left(m_{c}^{(\alpha)}(x, y)\right) & \left.=f_{c}^{-1}\left[1-\left(\alpha \cdot f_{c}(x)+(1-\alpha) \cdot f_{c}(y)\right)\right]=f_{c}^{-1}\left[\alpha \cdot\left(1-f_{c}(x)\right)+(1-\alpha) \cdot\left(1-f_{c}(y)\right)\right)\right] \\
& =m_{c}^{(\alpha)}\left(n_{c}(x), n_{c}(y)\right) .
\end{aligned}
$$

For $m_{d}$, the proof is similar.

\section{Aggregated equivalences}

Next, we define a new type of operator derived from the equivalences defined above. This new operator is a compromise between the normal and the dual equivalences (see Fig. 3), i.e. it fulfils neither $e(x, x)=1$ nor $e(x, n(x))=0$, but it has a nice property, namely $e(v, v)=v$. If we recall that the values represent uncertainities and $v$, as the fixpoint of the negation means that we hesitate whether the objects A and B have the particular property or not, it is also sensible to remain unsure about their equivalence value. This new operator will be called the aggregated equivalence operator.

Definition 18. The aggregated equivalence operators (see Fig. 4) are defined as follows.

$$
\begin{aligned}
& e_{c}^{*}(x, y)=m_{c}^{\left(\frac{1}{2}\right)}\left(e_{c}(x, y), \bar{e}_{c}(x, y)\right), \\
& e_{d}^{*}(x, y)=m_{d}^{\left(\frac{1}{2}\right)}\left(e_{d}(x, y), \bar{e}_{d}(x, y)\right) .
\end{aligned}
$$

Proposition 18. The aggregated equivalence operator in a bounded system

$$
e_{c}^{*}(x, y)=f_{c}^{-1}\left[\frac{1}{2}\left|f_{c}(x)-f_{c}(y)\right|+\frac{1}{2}\left(1-\left|f_{c}(x)+f_{c}(y)-1\right|\right)\right]
$$

and

$$
e_{d}^{*}(x, y)=f_{d}^{-1}\left[\frac{1}{2}\left(1-\left|f_{d}(x)-f_{d}(y)\right|\right)+\frac{1}{2}\left|f_{d}(x)+f_{d}(y)-1\right|\right] .
$$

Proof. Follows from direct calculation. 


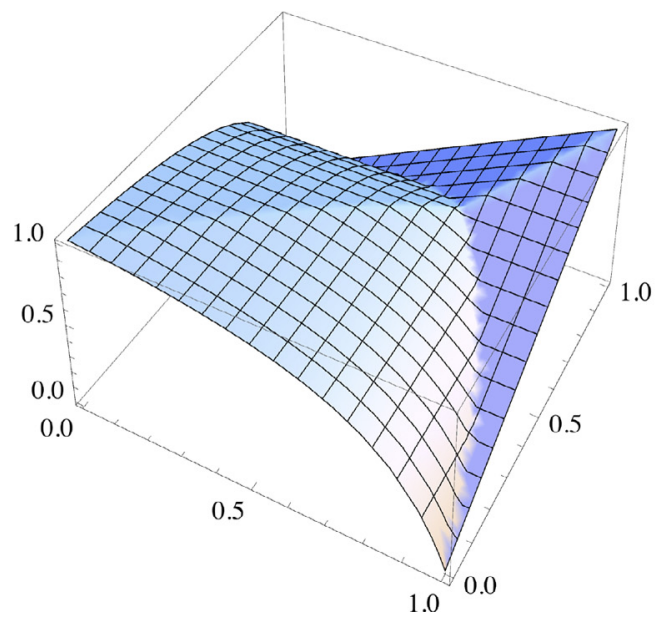

(a) $e_{c}^{*}$

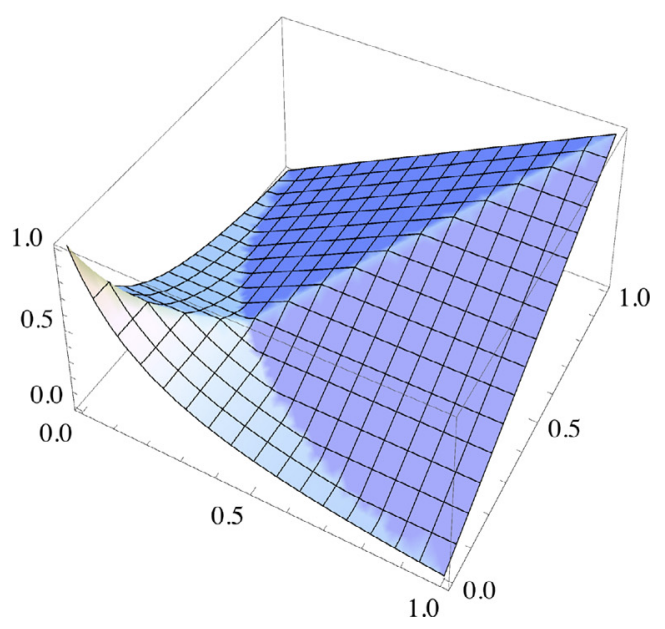

(b) $e_{d}^{*}$

Fig. 4. Aggregated equivalences with rational generators with $v=0.3$.

Proposition 19. The conjunctive and the disjunctive aggregated equivalence operators have the following property.

$$
\begin{gathered}
e_{c}^{*}(x, y)= \begin{cases}n_{c}(y), & \text { if } x \leq y \leq n_{c}(x) \\
x, & \text { if } n_{c}(y) \leq x \leq y \\
n_{c}(x), & \text { if } y \leq x \text { and } y \leq n_{c}(x) \\
y, & \text { if } y \leq x \text { and } y \geq n_{c}(x),\end{cases} \\
e_{d}^{*}(x, y)= \begin{cases}n_{d}(y), & \text { if } x \leq y \text { and } x \leq n_{d}(y) \\
x, & \text { if } n_{d}(y) \leq x \leq y \\
n_{d}(x), & \text { if } y \leq x \text { and } x \leq n_{d}(y) \\
y, & \text { if } y \leq x \text { and } n_{d}(y) \leq x .\end{cases}
\end{gathered}
$$

Proof. We prove for $e_{c}^{*}$. For $e_{d}^{*}$, the proof is similar.

1. If $x \leq y \leq n_{c}(x)$, then using the monotonicity of $f_{c}$ and the fact that $n_{c}(x)=f_{c}^{-1}\left(1-f_{c}(x)\right)$, we get $f_{c}(x) \geq$ $f_{c}(y)$ and $f_{c}(x)+f_{c}(y) \geq 1$. In this case it means that $e_{c}^{*}(x, y)=n(y)$.

2. If $n_{c}(y) \leq x \leq y$, then using the monotonicity of $f_{c}$ and the fact that $n_{c}(x)=f_{c}^{-1}\left(1-f_{c}(x)\right)$ we get $f_{c}(x) \geq f_{c}(y)$ and $f_{c}(x)+f_{c}(y) \leq 1$. In this case it means that $e_{c}^{*}(x, y)=x$.

3. If $y \leq x$ and $y \leq n_{c}(x)$, then we get $f_{c}(x) \leq f_{c}(y)$ and $f_{c}(x)+f_{c}(y) \geq 1$. In this case $e_{c}^{*}(x, y)=n_{c}(x)$ follows.

4. If $y \leq x$ and $y \geq n_{c}(x)$, then $f_{c}(x) \leq f_{c}(y)$ and $f_{c}(x)+f_{c}(y) \leq 1$. In this case it means that $e_{c}^{*}(x, y)=y$.

Next, we will examine the main properties of the aggregated equivalences. We will show that unlike the abovementioned equivalences, the aggregated equivalences are threshold transitive and associative as well.

Proposition 20. Let $v_{c}$ and $v_{d}$ be the fixpoints of $n_{c}$ and $n_{d}$, respectively. The aggregated equivalences have the following properties:

1. Compatibility (see Definition 1).

2. Symmetry (see Definition 1).

3. The aggregated equivalences are not reflexive, but $e_{c}^{*}\left(v_{c}, v_{c}\right)=v_{c}$ and $e_{d}^{*}\left(v_{d}, v_{d}\right)=v_{d}$ hold. In addition,

$$
e_{c}^{*}(x, x)= \begin{cases}n_{c}(x), & \text { if } x \leq v_{c} \\ x, & \text { if } x \geq v_{c}\end{cases}
$$


and similarly,

$$
e_{d}^{*}(x, x)= \begin{cases}n_{d}(x), & \text { if } x \leq v_{d} \\ x, & \text { if } x \geq v_{d}\end{cases}
$$

4. Monotonicity (see Definition 1).

5. $e_{c}^{*}$ is T-transitive with respect to the conjunction c (see Definition 2) and similarly, $e_{d}^{*}$ is T-transitive with respect to the $t$-norm generated by $1-f_{d}(x)$.

6. The aggregated equivalences are threshold transitive with respect to $v_{c}$ and $v_{d}$ (see Definition 2).

7. Invariance with respect to $n_{c}$ and $n_{d}$ (see Definition 2).

8. $e_{c}^{*}(1, x)=e_{d}^{*}(1, x)=x, e_{d}^{*}(0, x)=n_{d}(x)$, and similarly, $e_{c}^{*}(0, x)=n_{c}(x)$.

9. $e_{c}^{*}(x, y)=0$ if and only if $x, y \in 0,1$ and $x \neq y$. Similarly for $e_{d}^{*}$.

10. $n_{c}\left(e_{c}^{*}(x, y)\right)=e_{c}^{*}\left(n_{c}(x), y\right)$ if and only if $x \in\{0,1\}$ or $y \in\{0,1\}$ and $n_{d}\left(e_{d}^{*}(x, y)\right)=e_{d}^{*}\left(n_{d}(x), y\right)$ if and only if $x \in\{0,1\}$ or $y \in\{0,1\}$.

11. $e_{c}^{*}\left(x, v_{c}\right)=v_{c}$ and similarly, $e_{d}^{*}\left(x, v_{d}\right)=v_{d}$.

\section{Proof.}

1. Follows from direct calculation.

2. Trivial.

3. The statement follows from Proposition 18 and 19.

4. We show monotonicity for $e_{c}^{*}$. For $e_{d}^{*}$ the proof is similar. If $x \leq x^{\prime} \leq y^{\prime} \leq y$, then by Proposition 19 we have to consider two cases.

(a) $y \leq n_{c}(x)$. In this case $e_{c}^{*}(x, y)=n_{c}(y)$.

i. If $y^{\prime} \leq n_{c}\left(x^{\prime}\right)$, then $e_{c}^{*}\left(x^{\prime}, y^{\prime}\right)=n_{c}\left(y^{\prime}\right)$, which means that $e_{c}^{*}(x, y) \leq e_{c}^{*}\left(x^{\prime}, y^{\prime}\right)$.

ii. If $y^{\prime} \geq n_{c}\left(x^{\prime}\right)$, then $e_{c}^{*}\left(x^{\prime}, y^{\prime}\right)=x^{\prime}$ and $n_{c}(y) \leq n_{c}\left(y^{\prime}\right) \leq x^{\prime}$, so $e_{c}^{*}(x, y) \leq e_{c}^{*}\left(x^{\prime}, y^{\prime}\right)$.

(b) $y \geq n_{c}(x)$. In this case $e_{c}^{*}(x, y)=x$.

i. If $y^{\prime} \geq n_{c}\left(x^{\prime}\right)$, then $e_{c}^{*}\left(x^{\prime}, y^{\prime}\right)=x^{\prime}$, which means that $e_{c}^{*}(x, y) \leq e_{c}^{*}\left(x^{\prime}, y^{\prime}\right)$.

ii. If $y^{\prime} \leq n_{c}\left(x^{\prime}\right)$, then $e_{c}^{*}\left(x^{\prime}, y^{\prime}\right)=n_{c}\left(y^{\prime}\right)$ and $n_{c}\left(y^{\prime}\right) \geq x^{\prime} \geq x$, so $e_{c}^{*}(x, y) \leq e_{c}^{*}\left(x^{\prime}, y^{\prime}\right)$.

5. By using the decreasing property of $f_{c}^{-1}$ and the fact that $|a-b|-|a+b-1|+|b-c|-|b+c-1|+1 \geq$ $|a-c|-|a+c-1|$ holds for all $a, b, c \in[0,1]$, the statement follows from direct calculation. The proof is similar for $e_{d}^{*}$ as well.

6. We show the threshold transitivity for $e_{c}^{*}$. For $e_{d}^{*}$, the proof is similar.

The condition $e_{c}^{*}(x, y) \geq v_{c}$ is equivalent to the following inequality.

$$
f_{c}^{-1}\left[\frac{1}{2}\left|f_{c}(x)-f_{c}(y)\right|+\frac{1}{2}\left(1-\left|f_{c}(x)+f_{c}(y)-1\right|\right)\right] \geq v_{c}
$$

which means that

$$
\left|f_{c}(x)-f_{c}(y)\right| \leq\left|f_{c}(x)+f_{c}(y)-1\right| .
$$

This means that either $f_{c}(x), f_{c}(y) \leq \frac{1}{2}$, or $f_{c}(x), f_{c}(y) \geq \frac{1}{2}$ must hold, i.e. either $x, y \geq v_{c}$, or $x, y \leq v_{c}$.

Together with the condition $e_{c}^{*}(y, z) \geq v_{c}$, we also have that $y, z \geq v_{c}$, or $y, z \leq v_{c}$, from which we easily get that either $x, z \geq v_{c}$, or $x, z \leq v_{c}$ must hold, i.e. $e_{c}^{*}(x, z) \geq v_{c}$.

7. Follows from direct calculation.

8. Follows from the properties of $e_{c}, \overline{e_{c}}, e_{d}$, and $\overline{e_{d}}$.

9. The statement follows from Propositions 18 and 19.

10. Follows from direct calculation.

11. $e_{c}^{*}\left(x, v_{c}\right)=f_{c}^{-1}\left[\frac{1}{2}\left|f_{c}(x)-\frac{1}{2}\right|+\frac{1}{2}\left(1-\left|f_{c}(x)-\frac{1}{2}\right|\right)\right]=f_{c}^{-1}\left(\frac{1}{2}\right)=v_{c}$. Similarly for $e_{d}^{*}$ as well.

Remark 8. Note that from 3 , it follows immediately that $e_{c}^{*}(x, x) \geq v_{c}$ and similarly for $e_{d}^{*}$ as well.

Proposition 21. $e_{c}^{*}(x, y)>v_{c}$ if and only if $x, y>v_{c}$ or $x, y<v_{c}, e_{c}^{*}(x, y)=v_{c}$ if and only if $x=v_{c}$ or $y=v_{c}$, and $e_{c}^{*}(x, y)<v_{c}$ otherwise. Similarly for $e_{d}^{*}(x, y)$. 
Proof. The statement readily follows from Proposition 19.

Remark 9. Note that $e_{c}^{*}$ and $e_{d}^{*}$ considered as fuzzy binary relations on [0,1], are both c-transitive (see [16] p. 53).

Proposition 22. $e_{c}^{*}$ and $e_{d}^{*}$ are associative.

Proof. Let us consider $e_{d}^{*}(x, y)$. First, we will show that associativity holds in the case where $f_{d}(x)=1-x$. Let us use the following notation for the disjunctive aggregated equivalence for $f_{d}(x)=1-x$.

$$
L(x, y):=e_{d}^{*}(x, y)=\frac{1}{2}(|x+y-1|-|x-y|+1) .
$$

It can be shown that

$$
L(x, y)=\min (\max (1-x, y), \max (x, 1-y)) .
$$

From this, we get

$$
\begin{aligned}
L(x, L(y, z)) & =\min (\max (x, y, z), \max (x, 1-y, 1-z), \max (1-x, y, 1-z), \max (1-x, 1-y, z)) \\
& =L(L(x, y), z),
\end{aligned}
$$

which means that $L(x, y)$ is associative. In particular, for an arbitrary generator function $f_{d}$,

$$
f_{d}^{-1}\left(L\left(f_{d}(x), L\left(f_{d}(y), f_{d}(z)\right)\right)\right)=f_{d}^{-1}\left(L\left(L\left(f_{d}(x), f_{d}(y)\right), f_{d}(z)\right)\right)
$$

also holds. Since

$$
e_{d}^{*}(x, y)=f_{d}^{-1}\left[\frac{1}{2}\left(1-\left|f_{d}(x)-f_{d}(y)\right|\right)+\frac{1}{2}\left|f_{d}(x)+f_{d}(y)-1\right|\right]=f_{d}^{-1}\left(L\left(f_{d}(x), f_{d}(y)\right)\right),
$$

we have proved the associativity of $e_{d}^{*}(x, y)$. The proof for $e_{c}^{*}$ is similar as well.

Proposition 23. In a connective system, the above-defined equivalences $e_{c}^{*}(x, y)$ and $e_{d}^{*}(x, y)$ coincide if and only if $f_{c}(x)+f_{d}(x)=1$ (or equivalently $n_{c}=n_{d}$, i.e. in a Eukasiewicz system), where $f_{c}$ and $f_{d}$ are the normalized generation function of the conjunction and disjunction operators, respectively.

\section{Proof.}

1. If $f_{c}(x)+f_{d}(x)=1$, then using the fact that $f_{c}(x)=1-f_{d}(x)$ and $f_{c}^{-1}(x)=f_{d}^{-1}(1-x)$, we get $e_{c}^{*}(x, y)=$ $e_{d}^{*}(x, y)$.

2. If $e_{c}^{*}(x, y)=e_{d}^{*}(x, y)$, then in particular $e_{c}^{*}(0, x)=e_{d}^{*}(x, 0)$, which means that $n_{c}(x)=n_{d}(x)$ must hold for all $x \in[0,1]$.

\section{Conclusion}

In this paper, three different types of equivalence operators in bounded systems were studied. After taking a closer look at the implication-based equivalences, we examined the properties of the so-called dual equivalences. Using these two types of equivalence operators, a new concept of aggregated equivalences was introduced, which proved to possess nice properties like threshold transitivity, T-transitivity and associativity. The main properties of all the three types of the above-mentioned equivalence operators are summarized below (see Table 2).

Finally, for applications in image processing, we define the overall equivalence of two grey level images, and give an important semantic meaning to the aggregated equivalences.

In signal and image processing, the equivalence of two signals or two images is always of great importance.

Let us assume that two grey level images, i.e. two integer-valued function $f$ and $g$ defined on a subset $I^{2}$ of $\mathbb{Z}^{2}$, are given. After normalizing $f$ and $g$, the equivalence of the images can be calculated in each picture element $x$ of $I^{2}$ (pixel) by using the equivalence operators considered above. For simplicity, let us assume that $I=\{1, \ldots, n\}$. The overall equivalence of the two images (which measures the overlap) can be calculated by an arithmetic mean in the following way. 
Table 2

The main properties of equivalence operators.

\begin{tabular}{llll}
\hline & $\begin{array}{l}\text { Implication-based } \\
\text { equivalences } \\
e_{c}, e_{d}\end{array}$ & $\begin{array}{l}\text { Dual } \\
\text { equivalences } \\
\bar{e}_{c}, \bar{e}_{d}\end{array}$ & $\begin{array}{l}\text { Aggregated } \\
\text { equivalences } \\
e_{c}^{*}, e_{d}^{*}\end{array}$ \\
\hline Compatibility & $\checkmark$ & $\checkmark$ & $\checkmark$ \\
Symmetry & $\checkmark$ & $\checkmark$ & $\checkmark$ \\
Reflexivity & $\checkmark$ & - & - \\
$e(x, n(x))=0$ & - & $\checkmark$ & - \\
$e(v, v)=v$ & - & - & $\checkmark$ \\
Monotonicity & $\checkmark$ & - & $\checkmark$ \\
Threshold transitivity & - & - & $\checkmark$ \\
Invariance & $\checkmark$ & $\checkmark$ & $\checkmark$ \\
$e(1, x)=x$ & $\checkmark$ & $\checkmark$ & $\checkmark$ \\
$e(0, x)=n(x)$ & $\checkmark$ & $\checkmark$ & $\checkmark$ \\
Associativity & - & - & $\checkmark$ \\
T-transitivity & $\checkmark$ & $\checkmark$ & $\checkmark$ \\
\hline
\end{tabular}

Definition 19. Let us consider two normalized grey level images, $f, g: I^{2} \rightarrow[0,1]$, where $I=\{1, \ldots, n\}$. Their overall equivalence $E$ is defined the following way:

$$
E(f, g):=\frac{1}{n^{2}} \sum_{i, j=1}^{n} e(f(i, j), g(i, j)),
$$

where $e$ stands for one of the equivalences considered so far.

The overall equivalence can be defined for one dimensional signals similarly.

Note that for values around the middle grey level, the aggregated equivalences, $e_{c}^{*}$ and $e_{d}^{*}$, give the maximal level of uncertainty, which gives them an important semantic meaning. Therefore, when studying the equivalence of two grey level images, the aggregated equivalences are of great importance.

\section{Acknowledgements}

The authors are grateful to all anonymous referees whose comments and suggestions have significantly improved our original version of this paper. This study was partially supported by "ELITeam", TÁMOP-4.2.2.D-15/1/KONV2015-0024, on 'Quantitative Assessment of Macular Degeneration via Spectral-domain Optical Coherence Tomography'.

\section{References}

[1] M.I. Ali, F. Feng, M. Shabir, A note on $(\in, \in \vee q)$-fuzzy equivalence relations and indistinguishabilty operators, Hacet. J. Math. Stat. 40 (3) (2011) 383-400.

[2] M. Baczyński, B. Jayaram, Fuzzy Implications, Springer-Verlag, Berlin, Heidelberg, 2008.

[3] I. Beg, S. Ashraf, Fuzzy equivalence relations, Kuwait J. Sci. Eng. 35 (1A) (2008) 191-206.

[4] D. Boixader, J. Jacas, J. Recasens, Transitive closure and betweenness relations, Fuzzy Sets Syst. 120 (2001) 415-422.

[5] D. Boixader, On the relationship between T-transitivity and approximate equality, Fuzzy Sets Syst. 133 (2003) 161-169.

[6] M. Chakraborty, S. Das, On fuzzy equivalence 1, Fuzzy Sets Syst. 11 (1983) 185-193.

[7] M. Chakraborty, S. Das, On fuzzy equivalence 2, Fuzzy Sets Syst. 12 (1983) 299-307.

[8] I. Chon, $\in$-fuzzy equivalence relations, Kangweon-Kyungki Math. J. 14 (1) (2006) 71-77.

[9] B. De Baets, R. Mesiar, T-partitions, Fuzzy Sets Syst. 97 (1998) 211-223.

[10] M. De Cock, E. Kerre, On (un)suitable fuzzy relations to model approximate equality, Fuzzy Sets Syst. 133 (2003) 137-153.

[11] M. Demirci, J. Recasens, Fuzzy groups, fuzzy functions and fuzzy equivalence relations, Fuzzy Sets Syst. 144 (2004) $441-458$.

[12] J. Dombi, Towards a general class of operators for fuzzy systems, IEEE Trans. Fuzzy Syst. 16 (2008) 477-484.

[13] J. Dombi, Equivalence operators that are associative, Inf. Sci. 281 (2014) 281-294.

[14] J. Dombi, O. Csiszár, The general nilpotent operator system, Fuzzy Sets Syst. 261 (2015) 1-19.

[15] J. Dombi, O. Csiszár, Implications in bounded systems, Inf. Sci. 283 (2014) 229-240. 
[16] J.C. Fodor, M. Roubens, Fuzzy Preference Modelling and Multicriteria Decision Support, Kluwer, Dordrecht, 1994.

[17] M. Grabish, J. Marichal, R. Mesiar, E. Pap, Aggregation Functions, Cambridge University Press, New York, 2009.

[18] K.C. Gupta, R.K. Gupta, Fuzzy equivalence relation redefined, Fuzzy Sets Syst. 79 (1996) 227-233.

[19] B. Jayaram, R. Mesiar, I-fuzzy equivalence relations and I-fuzzy partitions, Inf. Sci. 179 (2009) 1278-1297.

[20] E. Klement, M. Navara, A survey on different triangular norm-based fuzzy logics, Fuzzy Sets Syst. 101 (1999) $241-251$.

[21] R. Mesiar, B. Reusch, H. Thiele, Fuzzy equivalence relations and fuzzy partitions, J. Mult.-Valued Log. Soft Comput. 12 (2006) 167-181.

[22] V. Murali, Fuzzy equivalence relations, Fuzzy Sets Syst. 30 (1989) 155-163.

[23] W.C. Nemitz, Fuzzy relations and fuzzy functions, Fuzzy Sets Syst. 19 (1986) 177-1191.

[24] M. Sabo, P. Strezo, On reverses of some binary operations, Kybernetika 41 (2005) 425-434.

[25] N. Schmechel, On the isomorphic lattices of fuzzy equivalence relations and fuzzy partitions, Mult. Valued Log. 2 (1996) 1-46.

[26] H. Thiele, N. Schmechel, On the mutual definability of fuzzy equivalence relations and fuzzy partitions, in: Proc. FUZZ-IEEE'95, Yokohama, Japan, 1995, pp. 1383-1390.

[27] R.T. Yeh, Toward an algebraic theory of fuzzy relational systems, in: Proc. Int. Cong. Cyber, Namur, Belgium, 1973, pp. 205-223.

[28] L.A. Zadeh, Fuzzy sets, Inf. Control 8 (1965) 267-274.

[29] L.A. Zadeh, Similarity relation and fuzzy ordering, Inf. Sci. 3 (1971) 177-200. 